\title{
Iron depletion by iron chelators or ferroportin inhibits HIV-1 through the induction of HIF1 $\alpha$, p21 and IKB $\alpha$ and the inhibition of CDK9 and CDK2
}

\author{
Namita Kumari', Dmytro Kovalsky², Denitra Breuer ${ }^{1}$, Xiaomei Niu', Sergei Nekhai ${ }^{1 *}$ \\ From Frontiers of Retrovirology: Complex retroviruses, retroelements and their hosts \\ Cambridge, UK. 16-18 September 2013
}

\section{Background}

Intracellular iron and oxygen levels regulate HIV-1 replication by affecting several steps in the HIV-1 life cycle including transcription [1]. Low oxygen levels and reduced cellular iron are inhibitory as CDK9/cyclin T1 and CDK2/ cyclin $\mathrm{E}$ activities are reduced and HIV-1 transcription is inhibited. The alpha subunit of hypoxia-induced factor 1 (HIF1 $\alpha)$ is stabilized under hypoxia and in the conditions of low cellular iron. Iron depletion by iron chelators or through the expression of ferroportin, an iron export protein, inhibits CDK2 and CDK9 activities and blocks HIV-1 transcription [1]. As neither CDK2 nor CDK9 requires iron for their enzymatic activity, an apparent upstream regulation is involved. Also, induction of heme oxygenase-1 (HO-1) by hemin was shown to inhibit HIV-1 [1] although the mechanism of the inhibition was not clarified. Here we analyzed the effect of iron chelators on HIV-1 transcription and replication and also analyzed the effect of heme, a condition present in sickle cell disease that may protect against HIV-1 infection [2].

\section{Results}

Novel iron chelators, PpY-eT and PpY-aT, efficiently inhibited HIV-1 and induced the expression of IkB $\alpha$, an inhibitor of NF-kB, that was not previously reported. The chelators also induce the expression of HIF1 $\alpha$, increased the expression of p21, inhibited enzymatic activity of CDK2 and shifted CDK9 from the large to the small complex. HIF1 $\alpha$ knockdown in promonocytic THP1 cells led to increased HIV-1 replication suggesting that HIF1 $\alpha$ may restrict HIV-1. Treatment with hemin induced both HO-1 and ferroportin expression and inhibited HIV-1. Hemin treatment also induced expression of IkB $\alpha$, HIF $1 \alpha$ and p21 thus mimicking the effect of iron chelators. Peripheral blood mononuclear cells obtained from patients with sickle cell disease showed increased expression of HO-1, ferroportin, IkB $\alpha$ and $\mathrm{p} 21$ and reduced ex-vivo HIV-1 replication.

\section{Conclusions}

HIV-1 transcription and replication is inhibited in low intracellular iron conditions which leads to the induction of IkB $\alpha$, HIF1 $\alpha$ and $\mathrm{p} 21$, resulting in inhibition of CDK2 and CDK9. The hemolytic conditions of sickle cell disease may stimulate ferroportin expression and intracellular iron reduction leading to the inhibition of HIV-1.

\section{Acknowledgements}

This project was supported by NIH Research Grants 1SC1GM082325, 2G12RR003048, and P3OHL107253.

\section{Authors' details}

${ }^{1}$ Center for Sickle Cell Disease, Howard University, Washington, DC, USA. ${ }^{2}$ ChemBio Center, National Taras Shevchenko University, Kiev, Ukraine.

Published: 19 September 2013

\section{References}

1. Nekhai S, Kumari N, Dhawan S: Role of cellular iron and oxygen in the regulation of HIV-1 infection. Future Virol 2013, 8:301-311.

2. Nouraie M, Nekhai S, Gordeuk VR: Sickle cell disease is associated with decreased HIV but higher HBV and HCV comorbidities in U.S. hospital discharge records: a cross-sectional study. Sex Transm Infect 2012, 88:528-533.

doi:10.1186/1742-4690-10-S1-P63

Cite this article as: Kumari et al:: Iron depletion by iron chelators or ferroportin inhibits HIV-1 through the induction of HIF1 $\alpha$, p21 and IKB $\alpha$ and the inhibition of CDK9 and CDK2. Retrovirology 2013 10(Suppl 1):P63.

${ }^{1}$ Center for Sickle Cell Disease, Howard University, Washington, DC, USA Full list of author information is available at the end of the article

(c) 2013 Kumari et al; licensee BioMed Central Ltd. This is an Open Access article distributed under the terms of the Creative Commons 